\title{
Point Process Formalism for Multiple Target Tracking
}

\author{
Shozo Mori \\ Information Extraction \& Transport (IET) \\ 1911 N. Fort Myer Dr., Suite 600 \\ Arlington, VA 22209 \\ Email: smori@iet.com
}

\begin{abstract}
This paper discusses the point process formalism of multiple target tracking problems. Finite point processes are defined as random elements in the spaces of finite sequences with their orders ignored, rather than as random integer-valued measures. Two recently developed algorithms, Joint Multitarget Probability (JMP) algorithm and Unified Data Fusion (UDF) algorithm will be described as algorithms based on the point process formalism. Two more established algorithms, Multiple Hypothesis Tracking (MHT) and Probabilistic Data Association (PDA) algorithms, will also be examined as algorithms using the point process concept. The point process formalism will then be compared with the random set formalism developed by Ron Mahler.
\end{abstract}

Keywords: Multiple Target Tracking, Point Process, Random Set, Joint Multitarget Probability, Unified Data Fusion, Multiple Hypothesis Tracking, Probabilistic Data Association

\section{Introduction}

Multiple target tracking problems concern tracking an unknown number of objects by multiple sensors that provide imperfect and noisy measurements, with possible misdetection and extraneous returns (false alarms) [1]-[5]. One of the unique characteristics of multiple target tracking problems is that a system state as well as each unit of sensor data ${ }^{1}$ is an array of elements in a given space such that (i) its length (size) is random, and (ii) its order does not contain any information. Mathematically, such characteristics can be expressed as random sets as defined in [6] and [7], which actually leads to the explicit use of random finite sets for multiple target tracking problems [8], [9]. However, there is another mathematical formalism that can be used to treat those characteristics, i.e., point processes, which we may say predate random sets [7], [10].

The objective of this paper is to describe general multiple tracking problems using the point process

\footnotetext{
1 Called measurement set in this paper, also called scan, frame, data set, etc.
}

\author{
Chee-Yee Chong \\ Booz Allen Hamilton \\ 101 California Street, Suite 3300 \\ San Francisco, CA 94111
}

Email: chong_chee@bah.com, cychong@alum.mit.edu

formalism, instead of the random set formalism, as described in [8], [9]. In particular, we will examine two recently developed multiple target tracking algorithms, Keith Kastella's Joint Multitarget Probability (JMP) algorithm [11], [12], and Larry Stone's Unified Data Fusion (UDF) algorithm [13], [14], as general multiple target tracking algorithms using the point process formalism. We will also re-examine two older algorithms, Multiple Hypothesis Tracking (MHT) algorithms [18], [19], and Probabilistic Data Association (PDA) algorithms [20], [21], from the same point of view. The point process formalism will later be compared with Ron Mahler's random set formalism [8].

In this paper, we assume that the target state space and the measurement spaces are hybrid spaces. By a hybrid space ${ }^{2}$ we mean the direct product of a Euclidean space and a finite set, together with the hybrid measure, i.e., the direct product measure of the Lebesgue measure on the Euclidean space and the counting ${ }^{3}$ measure on the finite set.

\section{Point process formalism}

Let $E=\Re^{d} \times\left\{s_{1},, s_{N}\right\}$ be a hybrid space with the hybrid measure $\mu$. Then $\bigcup_{n=0}^{\infty} E^{n}$ is the collection of all the finite sequences in $E$, where $E^{n}=\overbrace{E \times \cdots \times E}^{n \text { times }}$ is the set of all the sequences $\left(x_{i}\right)_{i=1}^{n}$ in $^{4} E$ with length $n$. For any

\footnotetext{
2 Use of the hybrid measure is for notational convenience to avoid the mixtures of summations and integrals. Theoretically, all the arguments made in this paper can be easily extended to the case where the state and measurement spaces are locally compact Housdorff spaces satisfying the second axiom of countability with a $\sigma$-finite measure.

${ }^{3}$ A counting measure $m$ on any measurable space $(E, B)$ is defined as, for any $B \in \mathbf{B}, m(B)=\#(B)$ if $B$ is a finite set, otherwise $m(B)=\infty$. $\#(A)$ is the cardinality of (the number of elements of) any set $A$.

${ }^{4}\left(x_{i}\right)_{i=1}^{n}$ is a shorthand of a finite sequence $\left(x_{1}, \ldots, x_{n}\right)$, while $\left\{x_{i}\right\}_{i=1}^{n}$ is a shorthand of a finite set $\left\{x_{1}, \ldots, x_{n}\right\}$ if $x_{1}, \ldots, x_{n}$ are all distinct, otherwise $\left\{x_{1}\right\}_{i=1}^{n}=\bigcup_{i=1}^{n}\left\{x_{i}\right\}$.
} 
element $x$ in $\bigcup_{n=0}^{\infty} E^{n}$, let $\ell(x)$ be the length of $x$, i.e., $\ell(x)=n \Leftrightarrow x \in E^{n}$. We define $E^{0}=\{\theta\}$ where $\theta \notin E$ is a special symbol for the "null sequence," and $\ell(\theta)=0$.

For each $n$, let $E^{n} / n$ ! be the quotient space induced on $E^{n}$ by equivalent classes [] defined by $\left\lfloor\left(x_{i}\right)_{i=1}^{n}\right\rfloor=\left\{\left(x_{a(i)}\right)_{i=1}^{n} \mid a \in A_{n}\right\}$ where $A_{n}$ is the set of all the permutations on $\{1, \ldots, n\}$, and let $\varphi: \bigcup_{n=0}^{\infty} E^{n} \rightarrow \bigcup_{n=0}^{\infty} E^{n} / n$ ! be the canonical map, i.e., $\varphi\left(\left(x_{i}\right)_{i=1}^{n}\right)=\left\lfloor\left(x_{i}\right)_{i=1}^{n}\right\rfloor$ for every $\left(x_{i}\right)_{i=1}^{n} \in E^{n}$. For the space $\bigcup_{n=0}^{\infty} E^{n} / n !$, consider the directsum topology of each quotient topology on $E^{n} / n$ !, and the measurable structure defined by that topology.

Then a finite point process in $E$ can be defined as a random element in $\bigcup_{n=0}^{\infty} E^{n} / n$ !, i.e., a finite point process in $E$ is the image $\varphi(x)=[x]$ of a random finite sequence $\boldsymbol{x}$ in $E$.

Suppose that the state space $E$ is finite (discrete), i.e., $d=0$, and let us ignore $\Re^{0}$ so that we have $E=\left\{s_{1}, \ldots, s_{N}\right\}$. For each $n$, define the canonical subset $\tilde{E}_{n}$ of $E^{n}$ by $\tilde{E}_{n}=\left\{\left(s_{i_{1}}, \ldots, s_{i_{n}}\right) \in E^{n} \mid i_{1} \leq i_{2} \leq \cdots \leq i_{n}\right\}$. Apparently, $\tilde{E}_{n}$ is isomorphic to $E^{n} / n$ !. Furthermore, assume that there is an a priori upper bound $\bar{n}$ on the length $\ell(x)$ of the random finite sequence $x$. Then the probability distribution of the finite point process $\varphi(x)=[x]$ can be defined as a probability measure $\Phi$ on $\bigcup_{n=0}^{\bar{n}} E^{n} / n !$ by $\Phi(\{[x]\})=\operatorname{Prob} .\{\varphi(x)=[x]\}$ for every $x \in \bigcup_{n=0}^{\bar{n}} \widetilde{E}_{n}$. Then we can define the probability density, $P: \bigcup_{n=0}^{\bar{n}} E^{n} / n ! \rightarrow[0, \infty)$, by $\Phi(\{[x]\})=P([x]) M(\{[x]\})$ for every $x \in \bigcup_{n=0}^{\bar{n}} \tilde{E}_{n}$, with respect to any given measure $M$ on $\bigcup_{n=0}^{\bar{n}} E^{n} / n$ ! such that $\left.{ }^{5} \quad M(\{x]\}\right)>0$ for every $x \in \bigcup_{n=0}^{\bar{n}} \tilde{E}_{n}$.

This probability density $P$, defined as a positive functional on a finite set $\bigcup_{n=0}^{\bar{n}} E^{n} / n$ !, is called Joint Multitarget Probabilities (JMP) in [11], [12] that describe Keith Kastella's JMP-based algorithm. In other words, in his model, an unknown number of targets is represented

\footnotetext{
${ }^{5}$ An obvious choice is $\left.M(\{x\}\}\right) \equiv 1$. Another choice, $M(\{x\})=(1 / n)) \mu^{n}\left(\varphi^{-1}(\{\varphi(x)\})\right)$ for every $x \in \tilde{E}_{n}$. With this choice, the functional $P$ can be called a Janossy density function, as shown in the next section.
}

by a finite point process on a finite set $E$. Larry Stone's Unified Data Fusion (UDF) algorithm described in [13], [14] uses a unique method for representing an unknown number of targets. In his model, the system state space is $(E \cup\{\theta\})^{n} / \bar{n}$ ! that is a quotient space induced from the direct-product space through the equivalent classes [] defined by the permutations of elements, where $\theta \notin E$ is a special symbol of "no-target." For example, $\left[\left(x_{1}, x_{2}, \theta, \ldots, \theta\right)\right] \in(E \bigcup\{\theta\})^{\bar{n}} / \bar{n}$ ! with $x_{1} \neq \theta$ and $x_{2} \neq \theta$ represents two targets at states $x_{1}$ and $x_{2}$. There is a oneto-one correspondence between $(E \bigcup\{\theta\})^{\bar{n}} / \bar{n} !$ and $\bigcup_{n=0}^{\bar{n}} E^{n} / n$ !, and hence, we can interpret Larry Stone's UDF algorithm also as the one that models an unknown number of targets as a finite point process.

Let another finite point process $[\boldsymbol{y}]$ in another finite set $^{6} E_{1}$ represent a random number of measurements taken at the same time from a given sensor. Then by extending the above arguments to the joint probability density $P([x],[y])$, the conditional probability density $P([y] \mid[x])$, etc., following the steps shown in [22], we can describe a model of a sensor which generates a measurement set $[\boldsymbol{y}]$ as

$$
\begin{aligned}
& P\left(\left[\left(y_{1}, \ldots, y_{m}\right)\right] \mid\left[\left(x_{1}, \ldots, x_{n}\right)\right]\right)= \\
& \sum_{a \in A(\{1, \ldots, n\},\{1, \ldots, m\})}\left(\prod_{i \in \operatorname{Dom}(a)} p_{M}\left(y_{a(i)} \mid x_{i} p_{D}\left(x_{i}\right)\right)\right) \\
& \left(\prod_{i \notin \operatorname{Dom}(a)}^{\prod}\left(1-p_{D}\left(x_{i}\right)\right)\right)\left(P_{F A}\left(\left[\left(y_{j}\right)_{j \notin \operatorname{Im}(a)}\right]\right)\right)
\end{aligned}
$$

for every $[y] \in \bigcup_{m=0}^{\infty} E_{1}^{m} / m$ ! and $[x] \in \bigcup_{n=0}^{\bar{n}} E^{n} / n$ !, where $p_{D}(x)$ is the target-wise independent probability of a target at $x$ being detected, $p_{M}(y \mid x)$ is the density of the target-wise independent target-state-to-measurement transition probability, and $P_{F A}$ is the probability density of a finite point process of independent false alarms contained in the measurement set $\left[\left(y_{1}, \ldots, y_{m}\right)\right]$. Then, the conditional probability density of the state $[x]$ as a finite point process given the measurement set $[\boldsymbol{y}]$ is given as $P([x] \mid[y])=P([y] \mid[x]) P([x]) / P([y])$.

\footnotetext{
${ }^{6}$ For JMP or UDF algorithm, measurement spaces do not have to be finite, as we assume here for simplicity.

${ }^{7}$ For any pair of sets, $(N, M), A(N, M)$ is the collection of all the oneto-one functions $a$ defined on a subset $\operatorname{Dom}(a)$ of set $N$ taking values in $M$. By $\operatorname{Dom}(a)$ and $\operatorname{Im}(a)$ we mean the domain and the image of any function $a$.
} 
Assuming conditional independence, the single-scan formula (1) can be easily extended to multiple-scan cases with measurement sets, $y(1), y(2), \ldots$, as finite point processes in the measurement spaces $E_{1}, E_{2}, \ldots$, using the filtering-update formula recursively. When the targets are dynamic, an appropriate Markov chain model can be constructed on the space $\bigcup_{n=0}^{\bar{n}} E^{n} / n$ !, based on the assumptions on the single-target dynamics.

Remark 1: Traditionally, a point process is defined as a random integer-valued (point) measure on a state space $E$, usually with measurability defined from a particular topology defined on a space of measures on $E$ (Cf., e.g., [29]). In fact, early works [15], [16] on multiple target tracking using the point process formalism use this random measure formulation. Since we need to use only finite point processes, it is much easier for us to treat a point process as a random sequence with random length as shown in [10]. According to [10], the use of the space $\bigcup_{n=0}^{\infty} E^{n}$ as a basis for finite point processes originates from [17]. A finite point process is a random finite sequence when its order is ignored. The only difference between a finite point process and a random finite set is existence or absence of repeated elements. When the state space contains a continuous component, it is not reasonable to allow repeated elements. On the other hand, when a target state space is quantized and becomes a discrete set, then it would be natural to allow repeated elements, as was done in this section, unless the cell size is extremely small.

\section{Multiple hypothesis tracking (MHT) algorithms}

As discussed in the previous section, a finite point process $[x]$ is an image $[x]=\varphi(x)$ of a random finite sequence $\boldsymbol{x}$

in $E$, i.e., a random element in $\bigcup_{n=0}^{\infty} E^{n}$. Hence, the probability distribution of a finite point process $[x]$ in hybrid space $E$ can be completely specified by, for each $n, p_{n}=\operatorname{Prob} .\{\ell(\boldsymbol{x})=n\}$ and a probability distribution $F_{n}$ on $E^{n}$ such that $F_{n}(B)=\operatorname{Prob}\{\boldsymbol{x} \in B \mid \ell(\boldsymbol{x})=n\}$ for every measurable set $B$ in $E^{n}$. For each $n$, for any collection of non-empty distinct compact sets $K_{1}, \ldots, K_{n}$ in $E$, we can write the event $\left\{[x] \in \varphi\left(\prod_{i=1}^{n} K_{i}\right)\right\}$ as the union of $n$ ! disjoint events $\left\{\boldsymbol{x} \in \prod_{i=1}^{n} K_{a(i)}\right\}$ with $n$ ! permutations $a$ on $\{1, \ldots, n\}$, and hence we have

$$
\begin{gathered}
\text { Prob. }\left\{[\boldsymbol{x}] \in \varphi\left(\prod_{i=1}^{n} K_{i}\right) \mid \ell(\boldsymbol{x})=n\right\}= \\
\sum_{a \in A_{n}} F_{n}\left(\prod_{i=1}^{n} K_{a(i)}\right)=n ! \tilde{F}_{n}\left(\prod_{i=1}^{n} K_{i}\right)
\end{gathered}
$$

where $\widetilde{F}_{n}$ is the scrambling or symmetrization of $F_{n}$, as defined as $\quad \tilde{F}_{n}\left(\prod_{i=1}^{n} K_{i}\right)=\frac{1}{n !} \sum_{a \in A_{n}} F_{n}\left(\prod_{i=1}^{n} K_{a(i)}\right)$. Apparently, each $\tilde{F}_{n}$ is permutable or symmetric in the sense that $\tilde{F}_{n}\left(\prod_{i=1}^{n} K_{a(i)}\right)=\tilde{F}_{n}\left(\prod_{i=1}^{n} K_{i}\right)$ for every $a \in A_{n}$.

For each $n$, a finite measure $J_{n}$ on $E^{n}$, defined by $J_{n}(B)=n ! p_{n} \widetilde{F}_{n}(B)$ for every measurable set $B$ in $E^{n}$, is called the $n$-th order Janossy measure $[10]^{8}$, and the density with respect to $\mu^{n}$, if it exists, is called the $n$-th order Janossy measure density and defined as $J D_{n}(x)=n ! p_{n} \tilde{f}_{n}(x)$ for every $x \in E_{n}$ where $\tilde{f}_{n}$ is the density of the permutable probability distribution $\tilde{F}_{n}$. We have

$$
\begin{gathered}
\text { Prob. }\left\{\left\{\boldsymbol{x}_{i}\right\}_{i=1}^{n} \cap K_{1} \neq \varnothing, \ldots,\left\{\boldsymbol{x}_{i}\right\}_{i=1}^{n} \cap K_{n} \neq \varnothing, \ell(\boldsymbol{x})=n\right\}= \\
J_{n}\left(K_{1} \times \cdots \times K_{n}\right)=n ! p_{n} \tilde{F}_{n}\left(K_{1} \times \cdots \times K_{n}\right)
\end{gathered}
$$

for any collection of non-empty distinct compact sets $K_{1}, \ldots, K_{n}$ in $E$.

In other words, a finite point process $[x]$ can be identified with a random finite sequence $\boldsymbol{x} \in \bigcup_{n=0}^{\infty} E^{n}$ when a permutable (symmetric) probability distribution $F_{n}$ is given for each $n$ as the joint distribution of $\boldsymbol{x}=\left(\boldsymbol{x}_{1}, \ldots, \boldsymbol{x}_{n}\right)$ when conditioned by $\ell(\boldsymbol{x})=n$. This is exactly the mathematical model used in [19] that, using hybrid spaces, generalized the Reid's MHT algorithm ([18]). In this sense, the results shown in [19] are based on the theory of the finite point processes.

By the definition of the canonical map as $\varphi(x)=[x]$ for every $x \in \bigcup_{n=0}^{\infty} E^{n}$, we have, for any measurable set $B$ in $E^{n}, \varphi^{-1}(\varphi(B))=\bigcup_{a \in A_{n}}\left\{\left(x_{a(i)}\right)_{i=1}^{n} \mid\left(x_{i}\right)_{i=1}^{n} \in B\right\}$, which we may call the scrambling or the symmetrization of set $B$. Then we can define the probability distribution $\Phi$ of the finite point process $[x]$ by

\footnotetext{
${ }^{8}$ According to [10], the term Janossy measure originated from [30] after its introduction in [31].
} 


$$
\begin{gathered}
\Phi\left(\varphi\left(\bigcup_{n=0}^{\infty} B_{n}\right)\right)=\text { Prob. }\left\{[x] \in \varphi\left(\bigcup_{n=0}^{\infty} B_{n}\right)\right\}= \\
\sum_{n=0}^{\infty} \frac{1}{n !} J_{n}\left(\varphi^{-1}\left(\varphi\left(B_{n}\right)\right)\right)=\sum_{n=0}^{\infty} p_{n} \tilde{F}_{n}\left(\varphi^{-1}\left(\varphi\left(B_{n}\right)\right)\right)
\end{gathered}
$$

for every collection $\left(B_{n}\right)_{n=0}^{\infty}$ of measurable sets such that each $B_{n}$ is a measurable set in $E^{n}$.

In parallel to eqn. (4), we can define a measure $M$ on $\bigcup_{n=0}^{\infty} E^{n} / n$ ! by

$$
M\left(\varphi\left(\bigcup_{n=0}^{\infty} B_{n}\right)\right)=\sum_{n=0}^{\infty} \frac{1}{n !} \mu^{n}\left(\varphi^{-1}\left(\varphi\left(B_{n}\right)\right)\right)
$$

for every $\left(B_{n}\right)_{n=0}^{\infty}$ such that each $B_{n}$ is a measurable set in $E^{n}$. Using this $\sigma$-finite measure $M$ on $\bigcup_{n=0}^{\infty} E^{n} / n$ !, we have

$$
\int_{\bigcup_{n=0}^{\infty} E^{n} / n !} \phi(X) M(d X)=\sum_{n=0}^{\infty} \frac{1}{n !} \int_{E^{n}} \phi(\varphi(x)) \mu^{n}(d x)
$$

for every non-negative measurable functional $\phi$ on $\bigcup_{n=0}^{\infty} E^{n} / n$ !.

Now assume that each $n$-th order Janossy measure $J_{n}$ has a density function $J D_{n}$ (the $n$-th order Janossy measure density). Using the Janossy measure densities, the probability distribution $\Phi$ of the finite point process $[x]$, can be written as

$$
\begin{aligned}
& \Phi\left(\varphi\left(\bigcup_{n=0}^{\infty} B_{n}\right)\right)=\text { Prob. }\left\{[x] \in \varphi\left(\bigcup_{n=0}^{\infty} B_{n}\right)\right\}=\sum_{n=0}^{\infty} \frac{1}{n !} J_{n}\left(\varphi^{-1}\left(\varphi\left(B_{n}\right)\right)\right) \\
& =\sum_{n=0}^{\infty} \frac{1}{n !} \int_{\varphi^{-1}\left(\varphi\left(B_{n}\right)\right)} J D_{n}\left(x_{1}, \ldots, x_{n}\right) \mu\left(d x_{1}\right) \cdots \mu\left(d x_{n}\right) \\
& =\int_{\varphi\left(\bigcup_{n=0}^{\infty} B_{n}\right)} P(X) M(d X)
\end{aligned}
$$

for every $\left(B_{n}\right)_{n=0}^{\infty}$ such that each $B_{n}$ is a measurable set in $E^{n}$, where the positive measurable functional $P$ on $\bigcup_{n=0}^{\infty} E^{n} / n$ ! is defined by

$$
P(\varphi(x))=P([x])=J D_{n}(x)
$$

for every $x \in \bigcup_{n=0}^{\infty} E^{n}$. We call this functional $P$ on $\bigcup_{n=0}^{\infty} E^{n} / n$ ! defined by (8) the Janossy density function that is nothing but the density of the probability distribution $\Phi$ of the finite point process $[x]$.
Let $\Gamma$ be a finite measure on a hybrid space $E$ with a density function $\gamma$ with respect to the hybrid measure $\mu$. Then a finite Poisson point process $[x]$ with an intensity measure $\Gamma$ is a finite point process that has a Janossy density function $P$ defined by $P\left(\left[\left(x_{i}\right)_{i=1}^{n}\right]\right)=e^{-v} \prod_{i=1}^{n} \gamma\left(x_{i}\right)$ for every $\left(x_{i}\right)_{i=1}^{n} \in E^{n}$ with $v=\Gamma(E)=\int_{E} \gamma(x) \mu(d x)<\infty$. Then $\gamma$ is called the intensity measure density of the Poisson point process $[x]$.

Let $[x]$ be the collection of targets modeled by a finite Poisson point process in a target state space $E$ with intensity measure density $\bar{\gamma}$ and let $[\boldsymbol{y}(1)], \ldots,[\boldsymbol{y}(k)]$ be the $k$ measurement sets, where each measurement set $[\boldsymbol{y}(\kappa)]$ is a finite point process in a measurement space $E_{\kappa}$. Each $[y(\kappa)]$ is defined by the conditional Janossy density function of eqn. (1), where the detection function $P_{D}$, the measurement transition probability density $P_{M}$, and the Janossy density function $P_{F A}$ for the finite point process modeling independent false alarms, are all indexed by measurement set index $\kappa$. Then a track is defined as a set of measurement indices $(j, \kappa)$ such that it selects at most one measurement index from one measurement set, and a (data association) hypothesis is defined as a collection of non-empty non-overlapping tracks.

Then, using the method similar to that used in [23], we can show that the conditional Janossy density function of the targets $[x]$ as a finite point process conditioned by conditionally independent measurement sets $[\boldsymbol{y}(1)], \ldots,[\boldsymbol{y}(k)]$ is expressed as

$$
\begin{aligned}
& P\left(\left|\left(x_{i}\right)_{i=1}^{n}\right| \mid[\boldsymbol{y}(1)], \ldots,[\boldsymbol{y}(k)]\right)= \\
& e^{-\hat{v}} \sum_{\lambda \in \Lambda} q(\lambda) \sum_{a \in \tilde{A}(\lambda,\{1, . ., n\})}\left(\prod_{\tau \in \lambda} f\left(x_{a(\tau)} \mid \tau\right)\right)\left(\prod_{i \notin \operatorname{Im}(\lambda)} \hat{\gamma}\left(x_{i}\right)\right)
\end{aligned}
$$

for every $\left(x_{i}\right)_{i=1}^{n} \in E^{n}$, where $\Lambda$ is the set of hypotheses, $(q(\lambda))_{\lambda \in \Lambda}$ is the evaluation of hypotheses such that $q(\lambda)>0$ for every $\lambda \in \Lambda$ and $\sum_{\lambda \in \Lambda} q(\lambda)=1, f(\cdot \mid \tau)$ is the density of the target state distribution conditioned by track $\tau$, and $\hat{\gamma}$ is the intensity measure density of the targets that are not detected by $([\boldsymbol{y}(1)], \ldots,[\boldsymbol{y}(k)])$ with $\hat{v}=\int_{E} \hat{\gamma}(x) \mu(d x)<\infty$.

\footnotetext{
${ }^{9}$ For any pair $(N, M)$ of sets, $\tilde{A}(N, M)$ is the collection of all the oneto-one function $a$ defined on $N(\operatorname{Dom}(a)=N)$ taking values in $M$ $(\operatorname{Im}(a) \subseteq M)$.
} 
Hypotheses can be generated as described in [18] and recursively evaluated using the generalized formula described in [19]. When the targets are dynamic and governed by a target-wise independent state transition probability density function, each track must be extrapolated between two measurement sets, assuming that they are sorted by time. Hypothesis evaluation formula can be expressed as a batch-processing form, from which the problem of seeking a best hypothesis $\lambda$ or a few best hypotheses in terms of its evaluation $q(\lambda)$ can be formulated and solved by the set of techniques, generally referred to as Multiple Dimensional Assignment or Multiple Frame Assignment algorithms [24] - [26]. Furthermore, track-oriented MHT algorithms [27], [28] are techniques in which tracks are recursively evaluated, but hypotheses are re-evaluated at the end of each measurement set processing cycle, using the recursively calculated track likelihood for each track.

Remark 2: The finite point processes used to define the target and the sensor models in this section are defined through the density functions. Therefore, if every hybrid space $E$ (or $E_{k}$ ) contains a Euclidean (continuous) space component (i.e., $E=\mathfrak{R}^{d} \times\left\{s_{1}, \ldots, s_{N}\right\}$ with $d>0$ ) so that every $\mu^{n}$ becomes atomless except for $n=0$, then the set of all the sequences with repeated elements has the product measure $\mu^{n}$ zero, i.e., $\mu^{n}\left(\left\{\left(x_{i}\right)_{i=1}^{n} \in E^{n} \mid x_{i}=x_{j}\right.\right.$ for some $\left.\left.i \neq j\right\}\right)=0$. The fact that we can ignore any repeated elements means that the model is equivalent to that described in the random finite set formalism discussed in Section 5. Nonetheless, this section shows that the general multiple hypothesis solution described in [19] can be formulated using general point processes formalism where repeated elements may be allowed.

\section{Tracking target with a priori identification: PDA}

In the previous two sections, targets are modeled by a finite point process. Assuming a separate track initialization mechanism, we may use a model in which the targets are given as an array of target states in a target state space $E$ with a fixed length and a fixed order. As mentioned in Section 3, if a given a priori joint probability distribution is permutable (symmetric), it is a special form of finite point process (with a fixed number of targets $p_{n}=1$ for some $n$ ). We assume that the joint probability distribution spatially separates the targets reasonably well, at least initially. In such a situation, we may call such targets targets with a priori identifications, in the sense that each target is identified a priori by its index ${ }^{10}$. In contrast, when the targets are modeled by a finite point process or a permutable joint probability distribution, the target indexing is meaningless in the sense that any one of the possible ways of indexing the targets is equally likely.

The same sensor model expressed by eqn. (1) can still be used after the condition, $\left[\left(x_{1}, \ldots, x_{n}\right)\right]$, for the conditional Janossy density function on the left hand side is replaced by the array $\left(x_{1}, \ldots, x_{n}\right)$ of target states in the target state space with a fixed length $n$. Since eqn. (1) is a linear combination of products of detection probabilities, measurement transition probability densities, and the false alarm Janossy density function, if the a priori target probability distribution is gaussian, and given each assignment $\quad a \in A(\{1, \ldots, n\},\{1, \ldots, m\}), \quad$ the sensor measurement transition can preserve gaussian-ness reasonably, then the "exact" solution, in terms of the joint probability distribution of the target states $\left(x_{1}, \ldots, x_{n}\right)$ conditioned by a series $([\boldsymbol{y}(1)] \ldots,[\boldsymbol{y}(k)])$ of measurement sets, each modeled by a finite point process, can be reasonably well approximated by a sum-of-gaussian distribution. However, it is apparent that the number of terms will explode as the number of measurement sets is accumulated.

The Probabilistic Data Association (PDA) algorithm [20] and its multiple target version, the Joint Probabilistic Data Association (JPDA) algorithm [21], are means for controlling the processing requirements under a fixed limit in all the circumstances.

The algorithm for processing one measurement set by a probabilistic association algorithm can be summarized as follows: (i) assume before processing each measurement set that the joint target state distribution is given as a set of independent gaussian distributions in a Euclidean space; (ii) generate all the assignment hypotheses as members of the set $A(\{1, \ldots, n\},\{1, . ., m\})$ with the number $n$ of targets and the number $m$ of measurements in the given measurement set; (iii) for each hypothesis, approximate the a posteriori probability distribution for each target as a gaussian distribution; (iv) combine all the hypotheses and approximate the joint target state distribution by a gaussian distribution by equating the mean vectors and the covariance matrices; and (v) approximate the joint gaussian distribution as the product of the independent probability distribution for each target.

\footnotetext{
${ }^{10}$ Larry Stone's UDF algorithm actually works on the target state space $(E \cup\{\theta\})^{\bar{n}}$. Therefore, if the joint a priori target state distribution is permutable, the algorithm generates $\bar{n}$ ! redundant solutions, effectively operating on the space $(E \cup\{\theta\})^{\bar{n}} / \bar{n}$ !. Otherwise, his algorithm can be viewed as the one with a priori target identifications.
} 


\section{Random set formalism}

Let I be the collection of all the finite sets in $E$. A random finite set is a random element in I. Redefine the $\operatorname{map} \varphi$ as a map from $\bigcup_{n=0}^{\infty} E^{n}$ to I by $\varphi\left(\left(x_{i}\right)_{i=1}^{n}\right)=\left\{x_{i}\right\}_{i=1}^{n}$ for every $\left(x_{i}\right)_{i=1}^{n} \in E^{n}$. This map $\varphi: \bigcup_{n=0}^{\infty} E^{n} \rightarrow 1$ is onto. It hence induces a topology and a measurable structure on I, inherited from $\bigcup_{n=0}^{\infty} E^{n}$. A random finite set can then be considered as an image $\varphi(x)$ of a random finite sequence $\boldsymbol{x}$ in $E$, i.e., a random element in $\bigcup_{n=0}^{\infty} E^{n}$. The equivalent classes generated by $\varphi: \bigcup_{n=0}^{\infty} E^{n} \rightarrow 1$ on $\bigcup_{n=0}^{\infty} E^{n}$ are coarser than the equivalent classes $[x]$ defined in Section 2, since we have $\varphi((x))=\varphi((x, x))=\varphi((x, x, x)) \cdots$ for any $x \in E$ while $[(x)] \neq[(x, x)]$. Otherwise, the equivalent class defined above and that defined in Section 2 both identify two sequences with different orders. Therefore, the only difference between a finite point process and a random finite set is that the former allows repeated elements while the latter does not. A point process without any repeated element is said to be simple. Hence we can say a random finite set is nothing but a simple finite point process. In fact, in some literature such as [7], no clear distinction between the two is made.

Let $\boldsymbol{X}$ be a random finite set in $E$, and in parallel to (3), define, for each $n$,

$$
\begin{aligned}
& J_{n}\left(K_{1} \times \cdots \times K_{n}\right)= \\
& \quad \text { Prob. }\left\{\boldsymbol{X} \cap K_{1} \neq \varnothing, \ldots, \boldsymbol{X} \cap K_{n} \neq \varnothing, \#(\boldsymbol{X})=n\right\}
\end{aligned}
$$

for any collection of non-empty distinct compact sets $K_{1}, \ldots, K_{n}$ in $E$. It is then appropriate to call a finite measure obtained by extending the definition (10) for any measurable sets in $E^{n}$ the $n$-order Janossy measure for random set $\boldsymbol{X}$. Let us call any measure on $E^{n}$ simple if it gives a measure zero to the set of all the sequences that contain a repeated element. Then the finite measure $J_{n}$ defined by (10) is always simple. We can re-define $\Phi$ in eqn. (4) as the probability distribution of random finite set $\boldsymbol{X}$ by

$$
\begin{gathered}
\Phi\left(\varphi\left(\bigcup_{n=0}^{\infty} B_{n}\right)\right)=\text { Prob. }\left\{X \in \varphi\left(\bigcup_{n=0}^{\infty} B_{n}\right)\right\}= \\
\sum_{n=0}^{\infty} \frac{1}{n !} J_{n}\left(\varphi^{-1}\left(\varphi\left(B_{n}\right)\right)\right)
\end{gathered}
$$

for every $\left(B_{n}\right)_{n=0}^{\infty}$ such that each $B_{n}$ is a measurable set in $E^{n}$. Conversely, if we are given a collection $\left(J_{n}\right)_{n=0}^{\infty}$ such that each $J_{n}$ is a simple finite measure on $E_{n}$ and $\sum_{n=0}^{\infty}(1 / n !) J_{n}\left(E^{n}\right)=1$, we can define uniquely a probability distribution $\Phi$ of a random finite set by (11).

Exactly the same equation (5) can be used with the different notation $\varphi\left(\left(x_{i}\right)_{i=1}^{n}\right)=\left\{x_{i}\right\}_{i=1}^{n}$ of the canonical map to define a $\sigma$-finite measure $M$ on the spacel of all the finite sets in $E$, and the integral with respect to this measure $M$ is now written as

$$
\int_{l} \phi(X) M(d X)=\sum_{n=0}^{\infty} \frac{1}{n !} \int_{E^{n}} \phi(\varphi(x)) \mu^{n}(d x)
$$

for every measurable positive functional $\phi$ on $\mathrm{I}$. Then eqn. (7) is re-written as

$$
\begin{aligned}
& \Phi\left(\varphi\left(\bigcup_{n=0}^{\infty} B_{n}\right)\right)=\text { Prob. }\left\{X \in \varphi\left(\bigcup_{n=0}^{\infty} B_{n}\right)\right\}=\sum_{n=0}^{\infty} \frac{1}{n !} J_{n}\left(\varphi^{-1}\left(\varphi\left(B_{n}\right)\right)\right) \\
& =\sum_{n=0}^{\infty} \frac{1}{n !} \int_{\varphi^{-1}\left(\varphi\left(B_{n}\right)\right)} J D_{n}\left(x_{1}, \ldots, x_{n}\right) \mu\left(d x_{1}\right) \cdots \mu\left(d x_{n}\right) \\
& =\int_{1} P(X) M(d X)
\end{aligned}
$$

for every $\left(B_{n}\right)_{n=0}^{\infty}$ such that each $B_{n}$ is a measurable set in $E^{n}$, where the positive measurable functional $P$ defined on I is defined by

$$
P\left(\varphi\left(\left(x_{i}\right)_{i=1}^{n}\right)\right)=P\left(\left\{x_{1}, \ldots, x_{n}\right\}\right)=J D_{n}\left(x_{1}, \ldots, x_{n}\right)
$$

for every finite set $\left\{x_{1}, \ldots, x_{n}\right\} \in \mathrm{I}$. We call this functional $P$ on I defined by (14) also the Janossy density function that is the density of the probability distribution $\Phi$ of the random finite set $\boldsymbol{X}$. As stated in the remark in the last section, the model used in the last section to describe a general MHT algorithm uses density functions. Therefore, if every hybrid space contains a Euclidean space, there is no repeated element in any of point processes used in the previous section, which justifies the use of the random set formalism to describe MHT algorithms in [23].

An MHT solution in the random set formalism can be written as the conditional Janossy density function $P$ of a random set $\boldsymbol{X}$ in the target state space $E$ that models the set of targets, conditioned by measurement sets, $\boldsymbol{Y}_{1}, \ldots, \boldsymbol{Y}_{k}$, modeled as conditionally independent random finite sets in measurement spaces $E_{1}, \ldots, E_{k}$,

$$
\begin{aligned}
& P\left(X \mid \boldsymbol{Y}_{1}, \ldots, \boldsymbol{Y}_{k}\right)= \\
& e^{-\hat{v}} \sum_{\lambda \in \Lambda} q(\lambda) \sum_{a \in \hat{A}(\lambda, X)}\left(\prod_{\tau \in \lambda} f(a(\tau) \mid \tau)\right)\left(\prod_{x \in X \backslash \operatorname{Im}(\lambda)} \hat{\gamma}(x)\right)
\end{aligned}
$$

with the same set of definitions for the set $\Lambda$ of hypotheses, hypothesis evaluation $q(\lambda)$, track evaluation $f(x \mid \tau)$, and the Janossy density function 
$P_{N D}(X)=e^{-\hat{v}} \prod_{x \in X} \hat{\gamma}(x)$ of a random set of the targets that are not detected in $\boldsymbol{Y}_{1}, \ldots, \boldsymbol{Y}_{k}$.

To our best knowledge, Ron Mahler is the first person who introduced the random finite set formalism, called Finite Set Statistics (FISST), for the multiple target tracking problems (Cf. e.g. [8]). He describes his mathematical foundation in [32]. He defines the integration of the functionals on the space I of all the finite sets in a given space $E$ by the right hand side of eqn. (12) and calls the integral the set integral. In his foundation, a functional $\beta$ on the collection of measurable sets $B$ in $E$, called the belief measure of the random set $\boldsymbol{X}$, is defined by $\beta(B)=\operatorname{Prob} .\{X \subseteq B\}$, and the density function ${ }^{11}$, i.e., the functional $P$ of (14), what we call the Janossy density function in this paper, is characterized as a form of the set-derivative of the belief measure.

In this paper, the probability distribution of a random finite set as well as a finite point process and its density function are derived through an equivalence relation on the space $\bigcup_{n=0}^{\infty} E^{n}$ of finite sequences in a given state space $E$, following the framework described in [10].

Remark 3: We may treat repeated elements as a part of a simple finite point process or a random finite set by extending the state space $E$ to $E \times\{1,2, \ldots\}$ or $E \times\{1, \ldots, m\}$ so that, e.g., $[(x, x)]$ in $E$ can be treated as $[((x, 2))](C f$., e.g., [29]). However, the advantage of such a formulation over that made in this paper is yet to be seen.

\section{Conclusion}

The point process formalism, in parallel to the random set formalism, of multiple target tracking problems, was described. It was shown that, for our purpose, the only essential difference between the point process and the random set formalisms is the potential presence or the absence of repeated elements, i.e., two targets occupying the same state, or two measurements with exactly the same value, etc. In practice, repeated elements make physical sense only when the state space is a finite set resulting from a quantization of a continuous space. We characterized the two recently developed multitarget tracking algorithms, JMP and UDF algorithms, as algorithms using the point process formalism, since these algorithms do allow two or more targets to occupy one

\footnotetext{
11 In the FISST terminology, $P(X), P(Y \mid X)$ and $P(X \mid Y)$ are called the global prior probability density function, the global likelihood function, and the global posterior probability density function, when $X$ is a random finite set of target states, and $Y$ is a random finite set of meeasurements.
}

cell, a single point in a state space. Two other older algorithms, MHT and PDA algorithms, were also reexamined using the point process formalism. In the past, when point processes were applied to multiple target tracking problems, they were introduced as random integer-values measures in a state space, as was customary in most literature on that subject. In this paper, a finite point process was treated as a random element whose realization is an equivalent class of finite sequences generated by ignoring the ordering of the elements in those sequences. A finite point process without repeated elements, i.e., a simple finite point process is essentially a random finite set.

It is the authors' opinion that, although the random set formalism (or the point process formalism) for multitarget tracking has provided a unified view on the subject of multiple target tracking, it has failed to produce any significant practical tracking algorithms, with the exception of the JMP and UDF algorithms examined in this paper. Another exception is the recent work ([33], [34]) by Ron Mahler on the use of moment measures, a concept developed in the point process framework, as the basis of a new kind of multiple target tracking algorithms. Once the relationship between point processes and random finite sets is clarified, we hope the point process framework will produce some more practical algorithms for multiple target tracking.

\section{References}

[1] Y. Bar-Shalom, and T.E. Fortmann, Tracking and Data Association, Academic Press, San Diego, 1988.

[2] Y. Bar-Shalom, and X. R. Li, Estimation and Tracking: Principles, Techniques and Software, Dedham, MA; Artech House, 1993 (Reprinted by YBS Publishing, Storrs, CT 1998).

[3] Y. Bar-Shalom, and X. R. Li, MultitargetMultisensor Tracking: Principles and Techniques, Storrs, CT, YBS Publishing, 1995.

[4] S. S. Blackman, Multiple-Target Tracking with Radar Application, Artech House, Norwood, MA, 1986.

[5] S. S. Blackman, and R. Popoli, Design and Analysis of Modern Tracking Systems, Artech House, Norwood, MA, 1999.

[6] G. Mathéron, Random Sets and Integral Geometry, John Wiley \& Sons, 1974.

[7] D. Stoyan, W.S. Kendall, and J. Mecke, Stochastic Geometry and its Applications, John Wiley \& Sons, 1987.

[8] R. Mahler, "Random Sets as a Foundation for General Data Fusion," Proceeding of the Sixth Joint Service Data Fusion Symposium, Vol. I, Part 1, John Hopkins University, Applied Physics Laboratory, Laurel, MD, pp. 357-394, 1993. 
[9] S. Mori, "Random Sets in Data Fusion: Multi-Object, State-Estimation as a Foundation of Data Fusion Theory," in Random Sets - Theory and Applications -, ed. By J. Goutsias, R.P.S. Mahler, and H.T. Nguyen, Springer Verlag, 1997.

[10] D. J. Daley, and D. Vere-Jones, An Introduction to the Theory of Point Processes, Springer-Verlag, 1988.

[11] K. Kastella, "Joint Multitarget Probabilities for Detection, Tracking, and Sensor Management," presented at Workshop on Applications and Theory of Random Sets, University of Minnesota, Minneapolis, MN, August 1996.

[12] K. Kastella, "Joint Multitarget Probabilities for Detection and Tracking," Proc. SPIE, Vol. 3086, pp. 122 $-128,1997$.

[13] C.A. Barlow, L. D. Stone, and M.V. Finn, "Unified Data Fusion," Proc. of the 9th National Symposium on Sensor Fusion, Monterey, CA, March 1996.

[14] L. D. Stone, C. A. Barlow, and T. L. Corwin, Bayesian Multiple Target Tracking, Artech House, 1999.

[15] R. B. Washburn, “A Random Point Process Approach to Multiobject Tracking," Proc. the 1987 American Control Conference, Minneapolis, MN, pp. 1846-1852, 1987.

[16] N. Portenko, H. Salehi, and A. Skorokhod, "On Optimal Filtering of Multitarget Tracking Systems Based on Point Processes Observations," Random Oper. and Stoch. Equ., Vol. 5, No. 1, pp. 1 - 34, 1997.

[17] J. E. Moyal, "The General Theory of Stochastic Population Processes," Acta. Math. Vol. 108, pp. 1 - 31, 1962.

[18] D. B. Reid, "An Algorithm for Tracking Multiple Targets," IEEE Trans. on Automat. Control, Vol. AC-24, No. 6, pp. 843-854, 1979.

[19] S. Mori, C-Y. Chong, E. Tse, and R.P. Wishner, "Tracking and Classifying Multiple Targets without $A$ Priori Identification," IEEE Trans. on Automat. Control, Vol. AC-31, No. 5, pp. 401-409, 1986.

[20] Y. Bar-Shalom, and E. Tse, "Tracking in a Cluttered Environment with Probabilistic Data Association," Automatica, Vol. 11, pp. 451-460, Sept. 1975.

[21] T.E. Fortmann, Y. Bar-Shalom, and M. Scheffé, "Multi-Target Tracking Using Joint Probabilistic Data Association," in Proc. 19th IEEE Conf. Decision Control, Albuquerque, NM, pp. 807-812, 1980.

[22] S. Mori, and C.-Y. Chong, "Theory of Multiple Target Tracking with a Review of Thirty Years of Multiple Target Tracking," Proc. Workshop on Estimation, Tracking and Fusion: A Tribute to Yaakov Bar-Shalom, Monterey, CA, May 2001.
[23] S. Mori, "Multi-Target Tracking Theory in Random Set Formalism," Proc. It International Conference on Information Fusion, Las Vegas, July 1998.

[24] S. Deb, M. Yeddanapudi, K. Pattipati, and Y. BarShalom, "A Generalized S-D Assignment Algorithm for Multisensor-Multitarget State Estimation," IEEE Trans. Aerospace and Electronic Systems, Vol. 29, No. 2, pp. 523-538, April 1997.

[25] R. L. Popp, K. R. Pattipati, and Y. Bar-Shalom, " $m$ Best SD Assignment Algorithm with Application to Multitarget Tracking," Proc. SPIE Conf. On Signal and Data Processing of Small Targets, Vol. 3373, Orlando, FL, April 1998.

[26] A. Poore, and N. Rijavec, "A New Class of Methods for Solving Data Association Problems Arising from Multitarget Tracking," Proc. 1991 American Automatic Control Conference, Vol. 3, pp. 2302-2304, Boston, MA, 1991.

[27] T. G. Allen, T. Kurin, and R. B. Washburn, "Parallel Computer Structures for Multiobject Tracking Algorithms on Associative Processors," Proc. American Control Conf., Seattle, WA, June, 1986.

[28] G. C. Demos, R. A. Ribas, T. J. Broida, and S. S. Blackman, "Application of MHT to Dim Moving Targets", 1990 Signal and Data Processing of Small Targets, Proc. SPIE, Vol.1305 (ed. O. E. Drummond), pp.297-309, April, 1990.

[29] A. F. Karr, Point Processes and Their Statistical Inference, Marcel Dekker, 1986.

[30] S. K. Srinivasan, Stochastic Theory and Cascade Processes, Academic Elsevier, New York, 1969.

[31] L. Janossy, "On the Absorption of a Nucleon Cascade," Proc. R. Irish Acad. Sci. Sec., Vol. A53, pp. 181-188, 1950.

[32] I. R. Goodman, R. P. S. Mahler, and H. T. Nguyen, Mathematics of Data Fusion, Kluwer, 1997.

[33] R. Mahler, "A Theoretical Foundation for the SteinWinter 'Probability Hypothesis Density (PHD)' Multitarget Tracking Approach," Proc. 2000 MSS National Symposium on Sensor and Data Fusion, San Antonio, TX, June, 2000.

[34] R. Mahler, "Multitarget Moments and Their Applications to Multitarget Tracking," Proc. Workshop on Estimation, Tracking and Fusion: A Tribute to Yaakov Bar-Shalom, Monterey, CA, May 2001. 